\title{
Pigments based on silica-coated gold nanorods: Synthesis, colouring strength, functionalisation, extrusion, thermal stability and colour evolution
}

\author{
Cyrille Gautier ${ }^{1}$, Alastair Cunningham ${ }^{2}$, Lynda Si-Ahmed ${ }^{1}$, Gilles Robert ${ }^{1}$ and Thomas Bürgi2 ${ }^{\star}$ \\ www.goldbulletin.org
}

\begin{abstract}
The intense plasmon absorption bands of gold nanorods (GNRs) with peak extinction coefficients up to $6.4 \times 10^{9} \mathrm{M}^{-1} \mathrm{~cm}^{-1}$ as well as their expected high stability make GNRs promising candidates for the colouration of bulk materials. The comparison of the integrated absorption in the visible region of GNRs with those of commercial organic pigments shows that the colouring strength of GNRs is 4 to 8 times higher.
\end{abstract}

In order to improve their stability, GNRs were encapsulated in a silica shell of around $15 \mathrm{~nm}$ thickness using an optimized Stöber method. The silica surface was modified with octadecylsilane to enable their dispersion in non-polar media. Different plastics were successfully coloured with a tiny quantity of bare and functionalised GNRs@SiO. These rods were homogeneously dispersed using extrusion. The shape of the rods was effectively stabilised by the silica shell at high temperature during the extrusion process. Surprisingly, a slight modification of the rods colour was observed due to a decrease of the refractive index in the mesoporous silica shell. However, this effect is greatly limited after the functionalisation.

\footnotetext{
Metalor Technologies SA, Avenue du Vignoble Case postale 9, CH-2009 Neuchâtel, Switzerland

Physikalisch Chemisches Institut, Ruprecht-Karls-Universität Heidelberg, Im Neuenheimer Feld 253, D-69120 Heidelberg, Germany

E-mail: buergi@uni-heidelberg.de

* Corresponding author: Prof. Dr. T. Bürgi, Physikalisch Chemisches Institut, Ruprecht-Karls-Universität Heidelberg, Im Neuenheimer Feld 253, D-69120 Heidelberg, Germany Fax: (+) 496221544918 E-Mail: buergi@uni-heidelberg.de
}

Title running head: GNRs@SiO 2 based pigment

Keywords: gold, silica, nanorods, thermal stability, porosity, coloring strength

\section{Introduction}

The plastic pigment market is considerable and a large portfolio of pigments is commercially available. However, the current pigment technology mainly based on metals, heavy metals and organics still suffers from major drawbacks ranging from low stability against heat, light and weather, poor migration resistance, difficulty of dispersion and strong impact on the physical properties of the polymers.

Furthermore, one has to mention the problem of toxicity of plastic containing environmentally unfriendly pigments (for example cadmium based yellow pigments). Finally, the pollution resulting from the plastic waste and recycling is also noteworthy, as pigmented plastic degradation leads to pollution with heavy metals or fragmented organic molecules. The development of more versatile pigments that will satisfy the majority of application requirements will help reduce the total amount of pigments used and simplify formulations.

Gold nanoparticles (GNPs) are extremely interesting pigment candidates. They were used as pigment of ruby-coloured stained glass dating back to the 17 th century. The red colouration of GNPs arises from their surface plasmon resonance (SP) due to the coherent oscillation of surface electrons when interacting with incident electromagnetic radiation.

Gold nanorods (GNRs) are fascinating nanomaterials with potential applications in various fields such as biosensing, [1-4] imaging, [5] therapy, [6] displays, [7] high-density optical storage, [8] patterning, [9] solar cells, [10] and spectrally selective coating for windows. [11] For GNRs, the SP resonance splits into two SP bands, the lateral $\left(S_{1}\right)$ and the longitudinal $\left(\mathrm{SP}_{\mathrm{L}}\right)$ surface plasmon resonances that correspond 
to electron oscillations perpendicular and parallel to the long rod axis, respectively.

GNRs are even more interesting pigment candidates than spherical GNPs. The strong dependence of the $S P_{L}$ on the aspect ratio (AR, length divided by width) of the GNRs allows fine-tuning of this absorption band from the visible to the infrared region. Hence, GNRs with different aspect ratios will have different colours, which is a unique property in pigment technology. SP resonance is very effective resulting in unrivalled extinction coefficient that can lead to a lower loading of pigment compared to the equivalent dyes used today in plastics. Indeed, the molar extinction coefficients for the maxima of the $\mathrm{SP}_{\mathrm{L}}$ band of GNRs were previously reported to be 4 orders of magnitude higher than those of commercial organic pigments.[12] These systems could then be used as dyes or pigments, affording many advantages over the organic dyes currently in use, not least of which includes the fact that GNRs are not photosensitive, ensuring colour stability over time. This is proven by examples such as the Lycurgus Cup, which was manufactured in Late Roman times and which still has a vibrant colour.[13] An additional advantage is that different colours can be produced relatively easily, simply by tuning the AR while it is often necessary to develop completely different synthetic routes to produce distinct colours using organic dyes.

We have exploited the well known Ag-assisted seedmediated synthesis for the preparation of GNRs.[14] We have compared the integrated absorption in the visible region of solutions containing GNRs and a commercial organic pigment at the same mass concentration. This shows that the colouring strength of the rods is 4 to 8 times higher. In order to improve their stability, GNRs were encapsulated in a mesoporous silica shell of around $15 \mathrm{~nm}$ thickness using an optimised Stöber method. The silica coating makes the GNR@SiO $\mathrm{Si}_{2}$ soluble in alcohols, allows the engineering of their surface via the versatile silica surface chemistry, improves their thermal stability and may preserve their colour after insertion in different media. The silica surface was modified with octadecylsilane (ODS) in order to disperse the GNRs@SiO 2 in non-polar media. Different plastics were successfully coloured with a tiny quantity of bare and functionalised GNRs@ $\mathrm{SiO}_{2}$ using a high volume manufacturing process (extrusion). The shape of the rods was effectively stabilised by the silica shell during extrusion at high temperature. Surprisingly, a slight modification of the rods colour was observed due to a decrease of the refractive index in the mesoporous silica shell. However, this effect is greatly limited after functionalisation. This study reveals the large potential of GNRs@SiO, as pigment for plastics and other bulk materials.

\section{Experimental procedures}

\section{Preparation of 4.2 aspect ratio GNRs@CTAB}

GNRs@CTAB, AR = $4.2 \pm 0.9$ were prepared according to procedures reported elsewhere. [14-17] Briefly, a growth solution was prepared by mixing at $27^{\circ} \mathrm{C}, 500 \mathrm{~mL}$ of a fresh $1 \mathrm{mM}$ aqueous chloroauric acid solution, $500 \mathrm{~mL}$ of CTAB (0.2 M) and $30 \mathrm{~mL}$ of a freshly prepared aqueous $\mathrm{AgNO}_{3}$ (4 mM) solution and $5.39 \mathrm{~mL}$ of $78 \mathrm{mM}$ Ascorbic acid solution. [18, 19] Concentrated chloroauric acid solution and silver nitrate powder were produced by Metalor.

Spherical gold seed particles were prepared by reducing $2.5 \times 10^{-4} \mathrm{M}$ aqueous chloroauric acid in $10 \mathrm{~mL}$ of $0.1 \mathrm{M} \mathrm{CTAB}$ at $27^{\circ} \mathrm{C}$ with $600 \mu \mathrm{L}$ of ice cold $\mathrm{NaBH}_{4}(0.001 \mathrm{M}$ in water) while the solution was stirred. A few minutes after the reduction with $\mathrm{NaBH}_{4}, 1.6 \mathrm{~mL}$ of this brownish yellow seed solution was added to the growth solution. The solution was left under gentle magnetic stirring for 3 hours.

GNRs@CTAB were separated from excess CTAB and unreacted gold and silver ions in solutions by centrifugation.

\section{Preparation of GNRs@SiO}

The pH of the clean GNRs@CTAB solution was adjusted to 10.5 through the addition of $\mathrm{NaOH}$ at 0.1 M. Subsequently, three additions of tetraethyl orthosilicate (TEOS, $4.23 \mathrm{~mL}, 20 \%$ in methanol) were introduced into the flask, at half-hour intervals. For each addition, the TEOS was added drop-wise and the reaction mixture was allowed to react for 12 hours. The AuNRs@SiO 2 were then centrifuged, and redispersed in ethanol.

\section{Preparation of GNRs@SiO_@ODS}

Surface modification with ODS was carried out following the procedure published by PastorizaSantos et al. [20] Briefly, $33.54 \mathrm{~mL}$ of ODS $(2.4 \%$ in chloroform) was added drop wise to $560 \mathrm{~mL}$ of AuNRs@SiO 2 (0.021 g.L.-1 in ethanol) with a pH adjusted to 10.5 with ammonia (25\%) and with vigorous stirring. After 24 hours, the particles were separated and washed by centrifugations in ethanol. 


\section{Results and discussion}

Preparation of GNRs@CTAB of $\mathbf{4 . 2}$ aspect ratio GNRs can be prepared using mainly four wet chemistry methods, the templated method, [21] the electrochemical method, [22] the photochemical method [23] and the seeded growth method. [10] In this study we used the silver-assisted seed mediated growth method which selectively gives access to GNRs with AR from 1 to 5 and thus to a wide panel of colour. [15] The shape and the optical properties of the rods are mainly controlled by the silver concentration in the growth solution. Furthermore the presence of silver enhances the selectivity towards the formation of GNRs up to $99 \%$. As shown by the TEM image in Figure 1, the rods have an averaged length and width of $50 \mathrm{~nm}$ and $12 \mathrm{~nm}$, respectively. These GNRs have an AR of $4.2 \pm 0.9$ and exhibit the two characteristic $\mathrm{SP}_{1}$ and $\mathrm{SP}_{\mathrm{L}}$ bands at around 520 and $820 \mathrm{~nm}$, respectively (see Figure 2). The $\mathrm{SP}_{\mathrm{L}} / \mathrm{SP}_{1}$ intensity ratio is superior to 3 showing that the proportion of sphere is negligible.

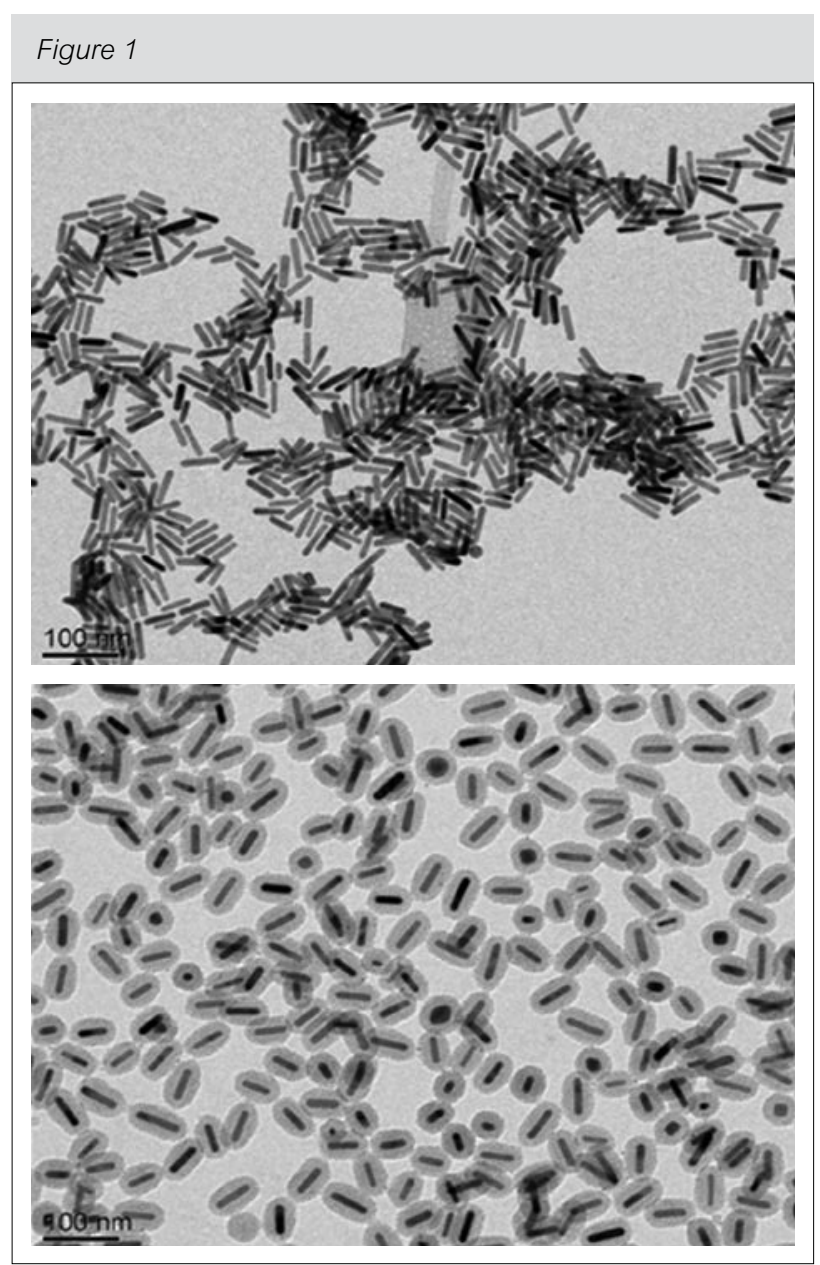

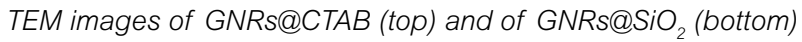

\section{Colouring strength}

El-Sayed et al. have published a systematic quantitative study which uses Mie theory and discrete dipole approximation method to calculate the trend of the absorption and scattering efficiencies for spherical nanoparticles, nanoshells and GNRs according to their diameter, to their $A R$ and to their volume. [24] This study clearly shows that GNRs are better candidates for pigmentation than nanospheres and nanoshells since a defined volume of gold in the rod shape gives higher absorption and scattering efficiencies. Their calculations are in good agreement with the extinction coefficients reported in literature and with our results. [25, 26] According to TEM, UVvis and inductively coupled plasma atomic emission, our GNRs exhibit a molar extinction coefficient of 6.4 $\times 10^{9} \mathrm{M}^{-1} \cdot \mathrm{cm}^{-1}$ at $820 \mathrm{~nm}$. Such extinction coefficients are at least 4 orders of magnitude higher than those of organic pigments. It is however difficult to compare the colouring strength of GNRs with those of commercial pigments based on extinction coefficients. Indeed, GNRs have very high molecular weight and their $\mathrm{SP}_{\llcorner}$bands are broader than typical absorption bands of organic dyes.

In order to assess the colouring strength of our brown GNRs, we have compared their integrated absorption in the visible region with the one of a commercial pigment (see Figure 3). It should be emphasized that this comparison is based on solutions having the same mass concentration of gold and dye, respectively. Cromophtal brown

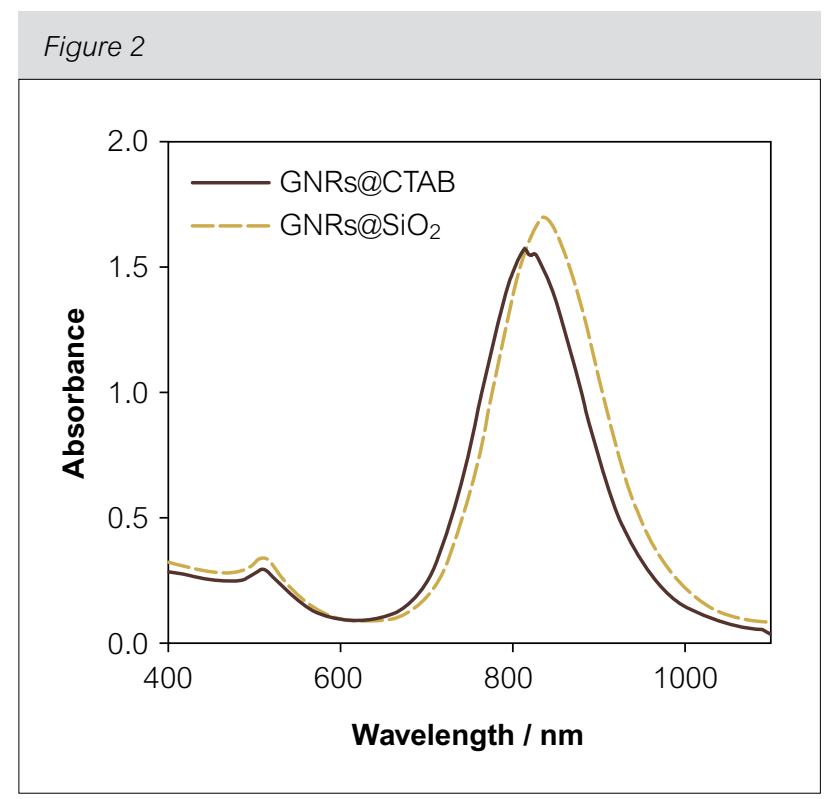

UV- vis spectra of GNRs@CTAB (solid black line) and

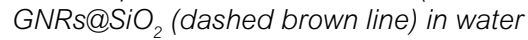




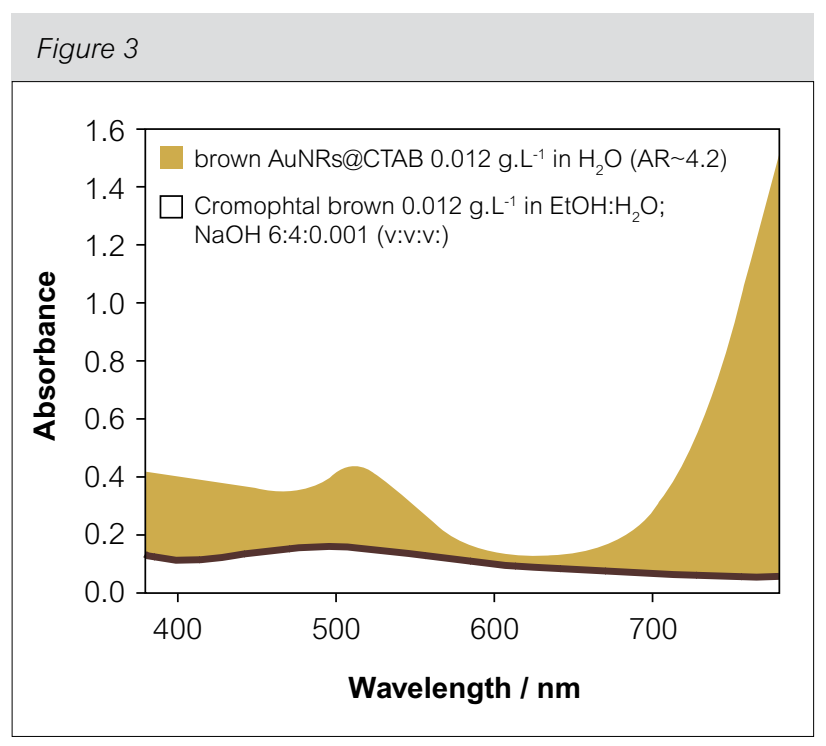

Visible spectra of GNRs@CTAB with AR $4.2\left(0.012\right.$ g.L L $^{-1}$ of gold in water, black line) and of Cromophtal brown (0.012 g. L $\mathrm{L}^{-1}$, EtOH: $\mathrm{H}_{2} \mathrm{O}: \mathrm{NaOH}, 6: 4: 0.001$, brown line). The brown surface shows the difference of absorption between brown GNRs@CTAB (AR 4.2) and Cromophtal brown

was selected because its La*b* coordinates are close to those of our GNRs. The result shows that the colouring strength or the integrated absorption of the rods is 3.7 higher even if a large part of the $\mathrm{SP}_{\perp}$ band is outside of the visible window. A similar comparison using gold nanorods with an AR of 2 and a blue colouration and Cromophtal blue shows that the colouring strength of the rods is up to 8 times higher due to the fact that the two plasmon bands are fully in the visible window.

\section{Preparation of GNRs@SiO}

The encapsulation of GNRs by oxide such as silica is expected to avoid the optical coupling of neighbouring GNRs, to preserve their colour upon environment modification, to protect the GNRs from aggregation, from thermal coalescence and from thermal rod-to-sphere shape transition. The latter was observed by increasing the temperature of the rods either in an oven or by laser irradiation. [27-29] Wang and co-workers have shown that the rod-tosphere shape transition induced by laser irradiation requires more energy when the rods are coated with silica. [27]

The well-known Stöber process is widely used for the formation of more or less dense spherical silica NPs by hydrolysis-condensation of TEOS in alkaline water/ethanol mixture. [30] The silica coating of AuNRs@SiO ${ }_{2}$ has been reported several times after removing the excess of CTAB by centrifugation

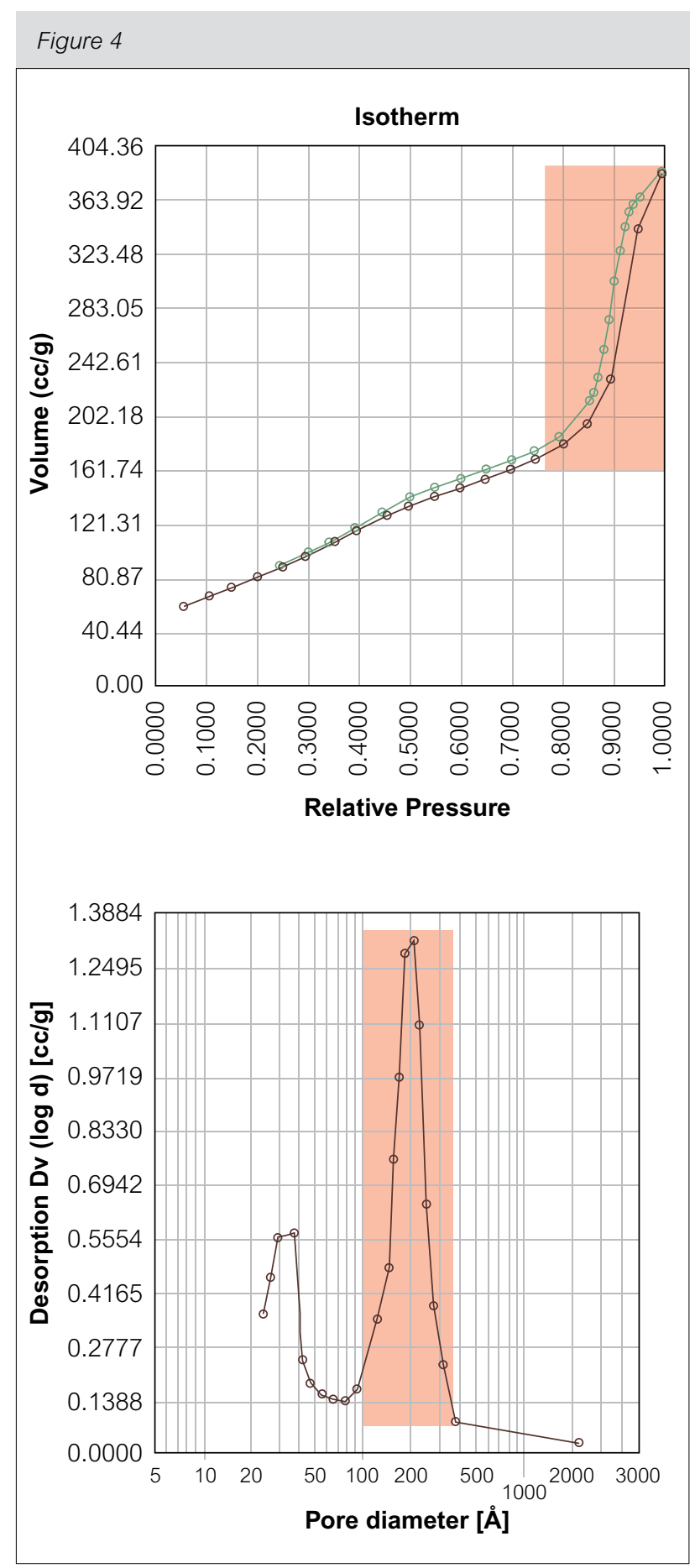

$N_{2}$ adsorption-desorption isotherm (top) and pore size distribution (bottom) of GNRs@SiO

either by direct hydrolysis-condensation of TEOS or sodium silicate on bare NRDs [31] or by using a primer to render the surface vitreophilic before silica coating. [27, 32-34] Both the direct hydrolysiscondensation method and the use of primer yield a silica shell with a uniform thickness over the entire 
surface of the GNRs. Furthermore, the silica shell thickness can be controlled with reaction time and reagent concentrations.

Here, GNRs@CTAB were coated using a direct improved Stöber method. Figure 1 shows a TEM image of the GNRs@SiO . It can be seen that the initial $A R$ is preserved during the coating, that the amorphous silica shell has a homogeneous thickness of ca. $15 \mathrm{~nm}$, and that the polymerisation of TEOS selectively takes place on the GNRs without visible agglomeration.

After the silica coating, the $\mathrm{SP}_{L}$ band is red shifted from $820 \mathrm{~nm}$ to $840 \mathrm{~nm}$ and the absorbance slightly increased due to the rise of the local refractive index around the GNRs (Figure 2). According to the results reported by Pastoriza-Santos et al., this $20 \mathrm{~nm}$ red shift is in good agreement with the thickness of 15 $\mathrm{nm}$ of the silica shell measured by TEM. [20]

The $\mathrm{N}_{2}$ adsorption-desorption isotherms (Figure 4) show that the silica shell obtained by this method is porous with a high surface area and a bimodal pore size distribution. Indeed, the surface area of the GNRs@SiO, is $303 \mathrm{~m}^{2} \cdot \mathrm{g}^{-1}$ and the curves show two well-defined adsorption steps. The first, at an intermediate relative pressure, is related to capillary condensation of $\mathrm{N}_{2}$ inside the silica shell mesopores which have a diameter of 3 to $4 \mathrm{~nm}$. The second step, at a high relative pressure, corresponds to the filling of the large meso- or macro-pores in between individual GNRs@SiO . In both cases, the curves show hysteresis due to a relatively wide pore size distribution as shown by the pore size distribution computed using the BJH treatment. [35] The metal core can be dissolved away to produce hollow silica nanotubes (using cyanide or aqua regia for gold or ammonia for silver). [32, 36] This porosity is likely due to the remaining CTAB micelles which are commonly used for the preparation of ordered mesoporous silica NPs. [37]

\section{Preparation of GNRs@SiO $@ O D S$}

In order to dissolve GNRs@SiO in non-polar media their surface was functionalised with ODS. The ODS

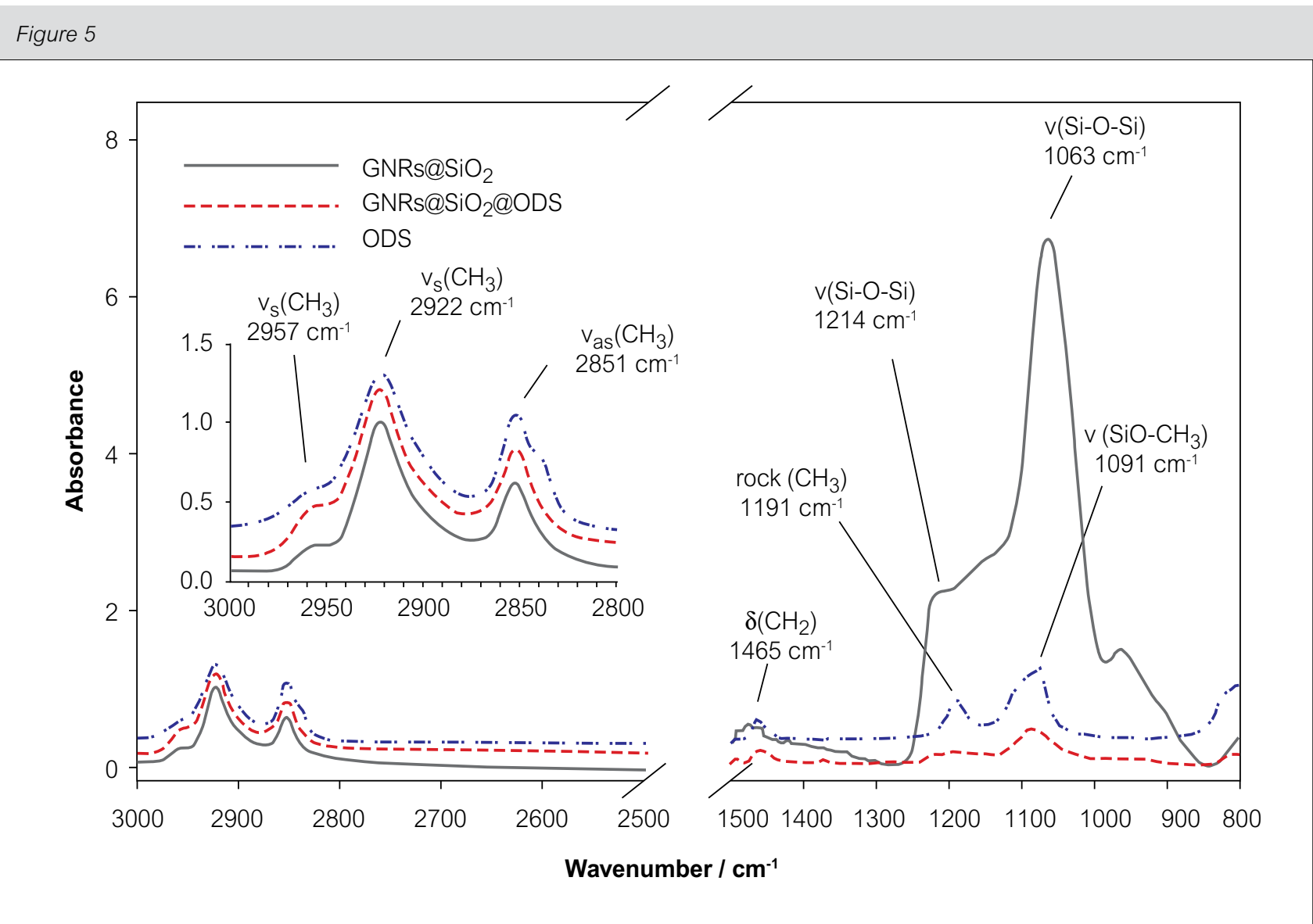

ATR-IR spectra of GNRs@SiO ${ }_{2}$ (black solid line), of GNRs@SiO @ODS (red dashed line) and of ODS (dash-dot-dot line) 
grafting was monitored by attenuated total reflection infrared spectroscopy (ATR-IR). As shown in Figure 5, ODS, GNRs@CTAB and GNRs@SiO_@ODS display characteristic bands of long alkyl chains. Additionally, ODS possesses bands at 1191 and $1091 \mathrm{~cm}^{-1}$ which can be assigned to the $\mathrm{CH}_{3}$ rocking and $\mathrm{SiO}-\mathrm{C}$ modes of $\mathrm{SiO}-\mathrm{CH}_{3}$, respectively. After the modification of the GNRs@SiO 2 with ODS, these bands were replaced by bands at 1214 and 1063 $\mathrm{cm}^{-1}$ which were assigned to the stretching modes of Si-O-Si. The grafting of ODS is clearly demonstrated by the fact that the Si-to-alkyl intensity ratio of related peaks decreases upon addition of ODS even after several centrifugations, by their solubility in nonpolar solvents and solid-state MAS NMR (Figure 6). The ${ }^{13} \mathrm{C}$ MAS NMR spectrum shows two signals centered at around 33 and 35 ppm which can be assigned to gauche and trans methylene
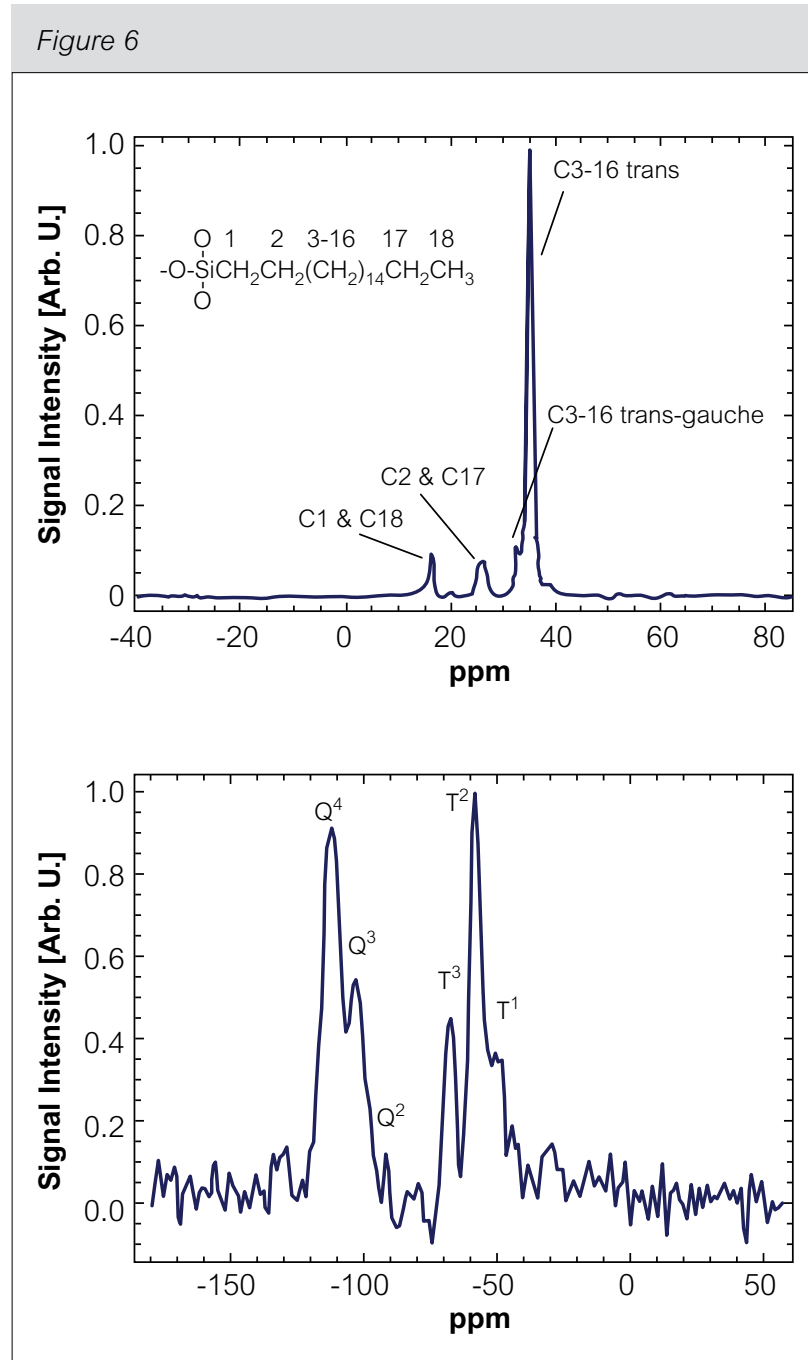

Solid-state MAS ${ }^{13} \mathrm{C}$ (top) and ${ }^{29} \mathrm{Si}$ (down) NMR spectra of GNRs@SiO_@ODS carbon conformations, respectively. Their relative intensity shows that ODS chains are mainly in trans conformation and that they are densely packed on the surface. The very small signal at 54.1 ppm shows that CTAB is almost completely removed.

The ${ }^{29} \mathrm{Si}$ MAS NMR spectrum shows mainly six peaks at around $-110,-100,-92,-65,-58$ and -50 ppm which can be assigned to $\mathrm{Q}^{4}\left(\mathrm{Si}(\mathrm{OSi})_{4}\right), \mathrm{Q}^{3}\left(\mathrm{Si}(\mathrm{OSi})_{3}(\mathrm{OH}\right.$ or $\mathrm{OR}), \mathrm{Q}^{2}\left(\mathrm{Si}(\mathrm{OSi})_{2}\left(\mathrm{OH}_{2}, \mathrm{OR}_{2}\right.\right.$ or $\left.(\mathrm{OH})(\mathrm{OR})\right), \mathrm{T}^{3}\left(\mathrm{RSiO}_{3}\right)$ and $\mathrm{T}^{2}\left(\mathrm{RSiO}_{2}(\mathrm{OH})\right)$ and $\mathrm{T}^{1}\left(\mathrm{RSiO}(\mathrm{OH})_{2}\right)$ silicon sites, respectively. Their relative intensities $0.9\left(\mathrm{Q}^{3}\right): 0.55$ $\left(\mathrm{Q}^{3}\right): 0.1\left(\mathrm{Q}^{2}\right): 0.45\left(\mathrm{~T}^{3}\right): 1\left(\mathrm{~T}^{2}\right): 0.35\left(\mathrm{~T}^{1}\right)$ reveal a high ODS coverage and a highly condensed framework. ${ }^{13} \mathrm{C}$ and ${ }^{29} \mathrm{Si}$ MAS NMR tend to show that both the surface and the pores of the silica shell are highly functionalised. The relatively high $\mathrm{T}^{2}$ signal might be attributed to not fully condensed ODS polymers either attached, or not, to the silica surface.

\section{Thermal stability}

The insertion of pigments in bulk material such as plastics often requires processes at high temperature such as extrusion and therefore the thermal stability of GNRs@SiO ${ }_{2}$ was investigated. It has been known for a long time that the melting point of a wide variety of materials such as metals, semiconductors and organic crystals decreases as their size is reduced to the nanometer scale. [38-44]

The rod shape of GNRs@CTAB was believed to be stable up to $\sim 600^{\circ} \mathrm{C}$. $[45,46]$ Figure 7 shows the UV-vis spectra of GNRs@CTAB dissolved in water, after drying in air at room temperature on a glass slide and after subsequent firing of this glass slide in air at $260^{\circ} \mathrm{C}$ for 90 minutes. After drying, the $\mathrm{SP}_{\mathrm{L}}$ band was broader and red shifted. This can be explained by interplasmon coupling due to the close proximity of the rods on the glass slide. Surprisingly, after heating the same glass slide for 90 minutes at only $260^{\circ} \mathrm{C}$, the $S_{L}$ band disappeared completely while the $S_{1}$ remained. This clearly demonstrates that a rod-tosphere shape transition occurs. The TEM image confirms this shape evolution. Petrova et al. and $\mathrm{Xu}$ and co-workers have already reported a similar transformation in the same range of temperatures for PVP coated and bare GNRs respectively. [11, 47] The formers also observed that a slow reduction of the AR is already observed at only $100^{\circ} \mathrm{C}$.

Silica coating of spherical gold NPs has been proven to be a very efficient approach to preserve the identity (volume) of the NPs even at high temperature. [29] Silica has a high melting temperature $(\sim 1700 \mathrm{~K})$ 


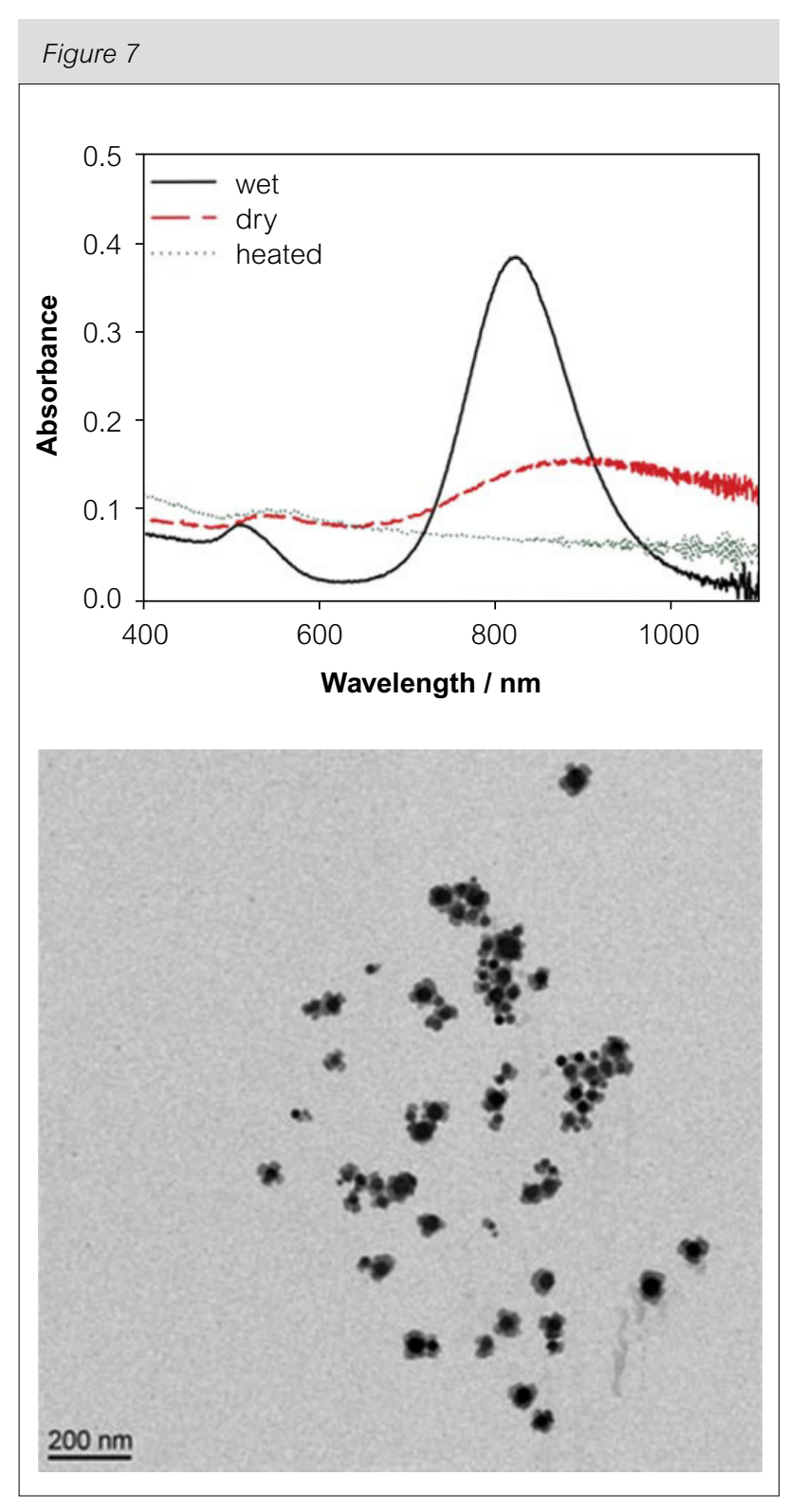

UV-vis spectra (top) of GNRs@CTAB in water (solid black line), on glass after drying in air at room temperature (red dashed line) and after heating at $260^{\circ} \mathrm{C}$ for 90 minutes (dotted green line). TEM image (bottom) of GNRs@CTAB after 90 minutes at $260^{\circ} \mathrm{C}$

and the silica shell of silica coated GNRs is known to withstand the GNRs reshaping induced by laser irradiation without deformation. [8]

In a similar way, the thermal stability of ODSfunctionalised and of bare GNRs@SiO, at $260^{\circ} \mathrm{C}$ was investigated. In both cases, interplasmon band coupling was not observed since the $15 \mathrm{~nm}$ shell is thick enough. However, a surprising colour change was observed when the GNRs@SiO 2 were dried in air on a glass substrate at room temperature. The UV-

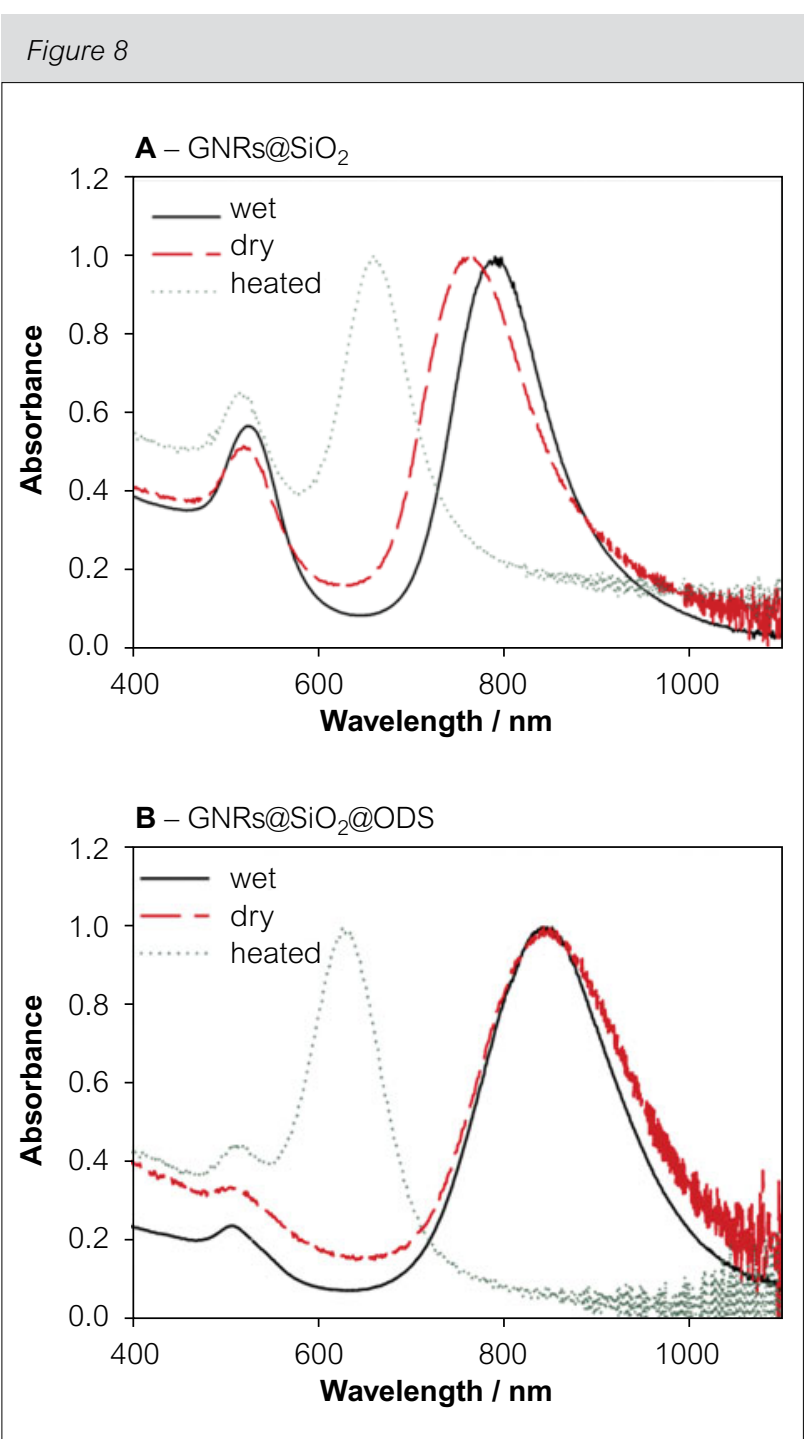

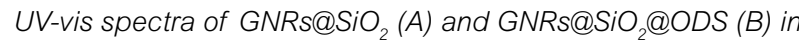
water (solid black line) and on glass slide after drying in air at room temperature (red dashed line) and after heating at $260^{\circ} \mathrm{C}$ for 90 minutes (dotted green line)

vis spectrum of the $\mathrm{GNR}_{\mathrm{S}} \mathrm{SiO}_{2}$ film indicates that the $S_{L}$ shifts from $802 \mathrm{~nm}$ to $774 \mathrm{~nm}$ during the drying process (see Figure 8-A). This can be attributed to the decrease of the refractive index when changing the GNR surrounding from ethanol to air. Wang et al. already observed this phenomenon for GNRs@SiO . [48] They observed that this colour change is highly reversible when the rods are repeatedly dried and dispersed in ethanol. This is in good agreement with the porosity of the silica shell discussed above.

Interestingly, in the case of the GNRs@SiO $@ 0 O D S$ (Figure 8-B), we observed that the $\mathrm{SP}_{\mathrm{L}}$ did not shift upon drying at room temperature. This might be due to the presence of the ODS chains on the surface and 
in the pores of the shell which does not evaporate upon drying and which stabilises the refractive index in the silica shell.

Upon heating at $260^{\circ} \mathrm{C}$ for 90 minutes, the $\mathrm{SP}_{\mathrm{L}}$ of both GNRs@SiO and GNRs@SiO @ODS are shifted from $774 \mathrm{~nm}$ to $662 \mathrm{~nm}$ and from $851 \mathrm{~nm}$ to $632 \mathrm{~nm}$, respectively. These blue shifts are comparable to those observed after insertion in plastics (vide infra). In the case of the GNRs@SiO_@ODS, the strong blue shift of the $S P_{L}$ band observed after heating above the ODS melting point might be attributed to the loss of the grafted ODS.

The TEM images of GNRs@SiO observed on the same grid at the same position before and after heating at $260^{\circ} \mathrm{C}$ for 90 minutes reveal that the shape of the rods is well preserved by the silica shell which may act as a mould.

\section{Colouration of plastics by gold GNRs@SiO ${ }_{2}$ and GNRs@SiO_@ODS}

Polycarbonate (PC) and polystyrol (PS) were coloured by both GNRs@SiO ${ }_{2}$ and GNRs@SiO $@$ ODS. An ethanol solution of the corresponding rods was added in a drop-wise fashion to the desired plastic pellets during the extrusion process at $260^{\circ} \mathrm{C}$. The initially uncoloured PS and PC are clearly tinted after insertion of the GNRs (see Figure 9). The concentrations of gold in PS doped with GNRs@ $\mathrm{SiO}_{2}$ and with GNRs@SiO $@$ ODS and in PC doped with GNRs@SiO 2 and with GNRs@SiO $@ O D S$ were $0.01,0.008,0.006$ and 0.002 wt. \%, respectively.

The initial GNRs@SiO 2 and GNRs@SiO $@ @ O D S$ solutions had almost the same brown colour. However, despite the silica coating, the colours of the doped plastics are different depending on the plastic and above all on the GNR surface. The UVvis spectra (Figure 10) of the pure plastics do not exhibit any structure whereas the spectra of the rod solutions and of the doped plastics clearly show the two characteristic SP bands. On the one hand, the $\mathrm{SP}_{L}$ to $S \mathrm{P}_{1}$ intensity ratios ( 3) and band width are well preserved after extrusion meaning that the sphere to rod proportions did not increase and that the rods are well dispersed after extrusion. On the other hand, the $S P_{L}$ bands were blue shifted whereas the $\mathrm{SP}_{1}$ band did not move. Importantly, it should be noted that the $S P_{L}$ blue shift is more significant for AuNRs@SiO 2 than for AuNRs@SiO 20 ODS.

For TEM analysis, the plastic pellets doped with the GNRs were cut into thin sections of around $100 \mathrm{~nm}$ using an ultramicrotome. The TEM observations (Figure 11) show that AuNRs@SiO 2 and NRs@ $\mathrm{SiO}_{2} @ \mathrm{ODS}$ are well dispersed in plastics and that their shape and their silica shells are well preserved during the extrusion process. However the AR cannot be measured due to the random orientation and to the low concentration of GNRs in the plastics.

According to Mie theory, the $\mathrm{SP}_{L}$ blue shifts can be attributed either to an $A R$ reduction or to a decrease of the dielectric constant of the GNRs environment during the extrusion process. Combining the information from TEM, $\mathrm{N}_{2}$ adsorption-desorption isotherms and UV-vis spectroscopic analysis, it is clear that the observed colour change is due to a modification of the dielectric constant in the GNRs environment. As verified above, the silica shell is stable enough to preserve the shape of the GNRs during the extrusion process. A very likely explanation for the colour change is consequently the evaporation of ethanol in the pores of the silica shell upon heating during the extrusion process leading to a refractive index decrease around the GNRs. This evaporation is also in good agreement with the limited blue shift in the case of the GNRs@SiO $@ O D S$ since the ODS molecules are strongly bonded and thus maintain a higher refractive index in the silica shell.

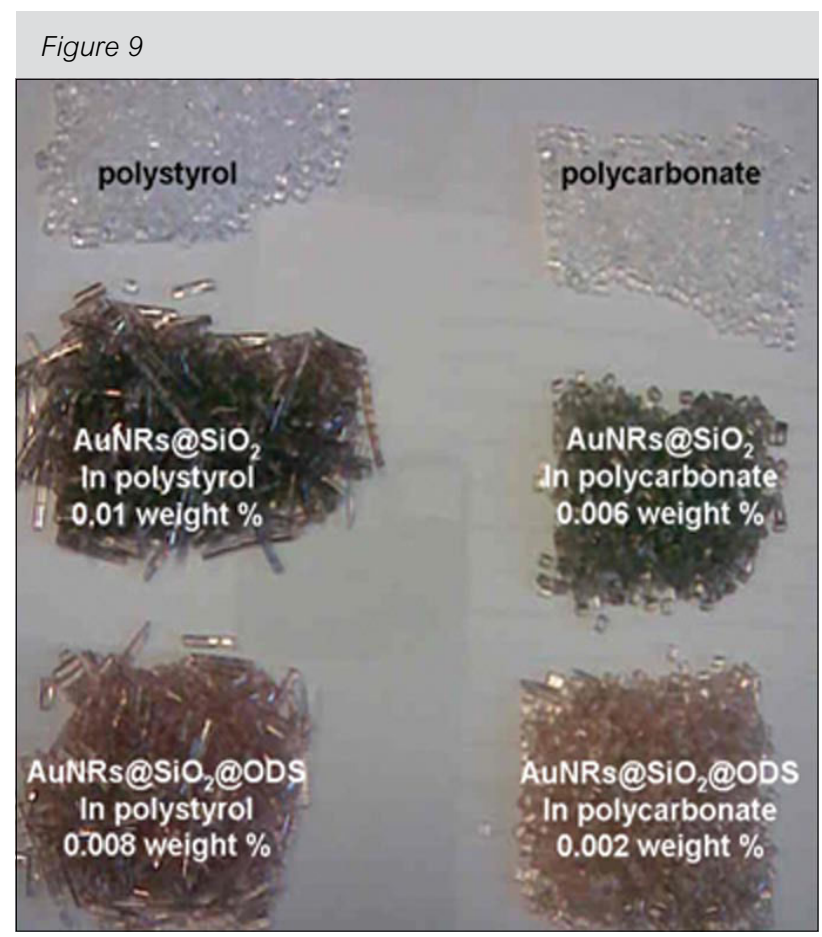

Picture of the pure plastic pellets (polystyrol and polycarbonate) and of the doped plastic pellets using GNRs@SiO ${ }_{2}$ and GNRs@ $\mathrm{SiO}_{2} @ \mathrm{ODS}$ with $\mathrm{AR} \sim 4.2$ 

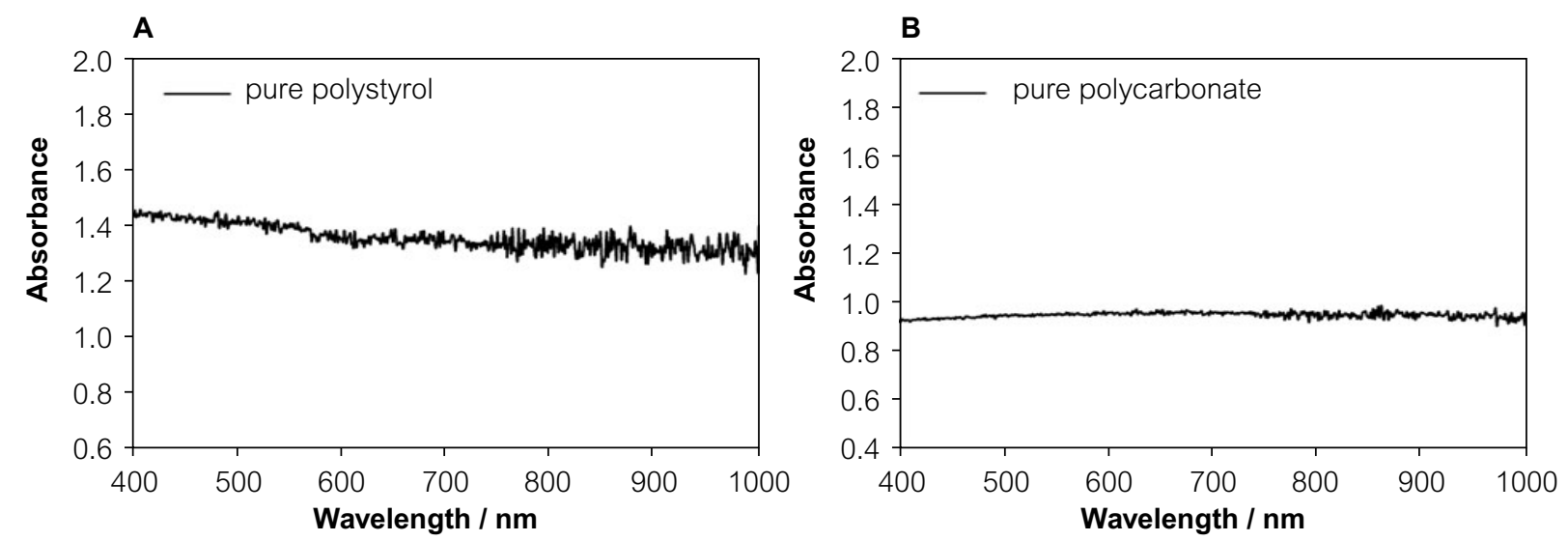

C

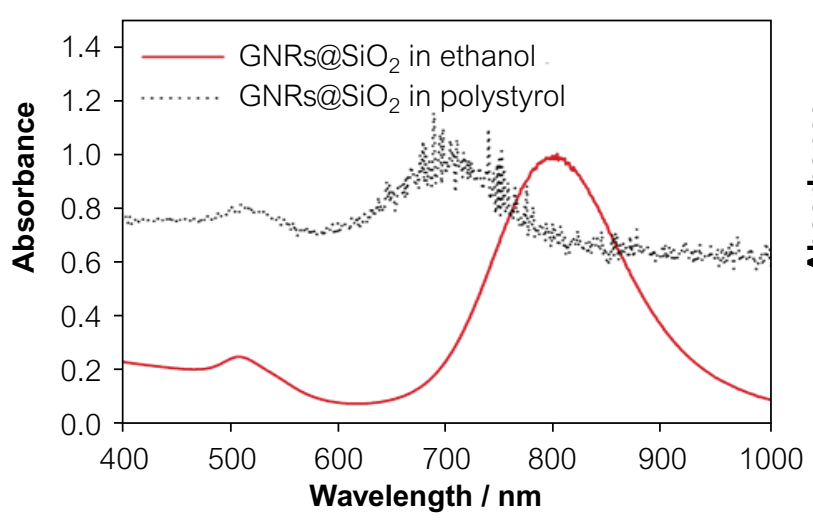

$\mathrm{E}$

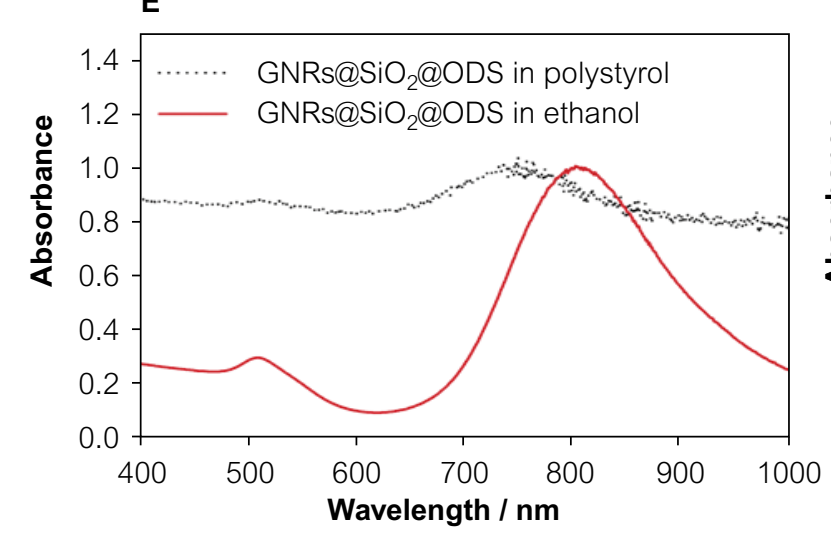

D
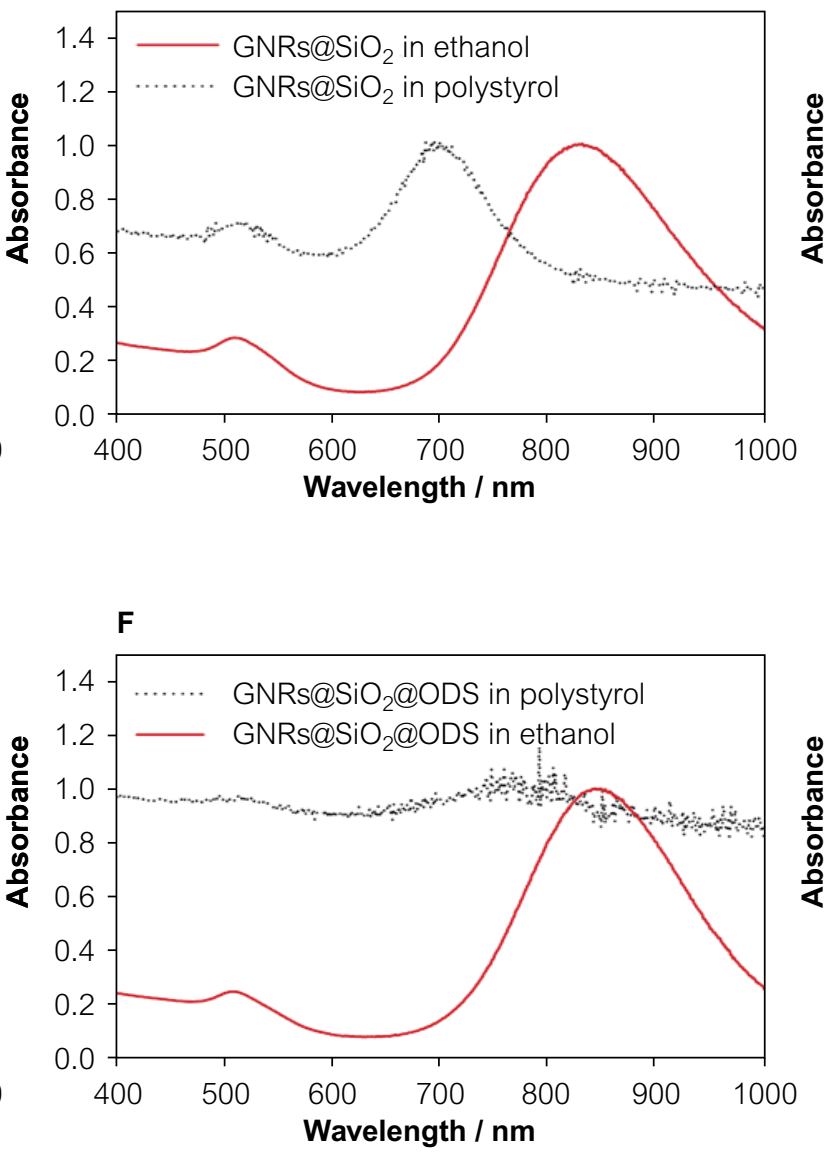

UV-vis spectra of the pure polystyrol and polycarbonate pellets (solid black lines), of the GNRs@SiO ${ }_{2}$ and GNRs@SiO @ODS in solution (red solid lines) and included in plastic (dotted black lines) 


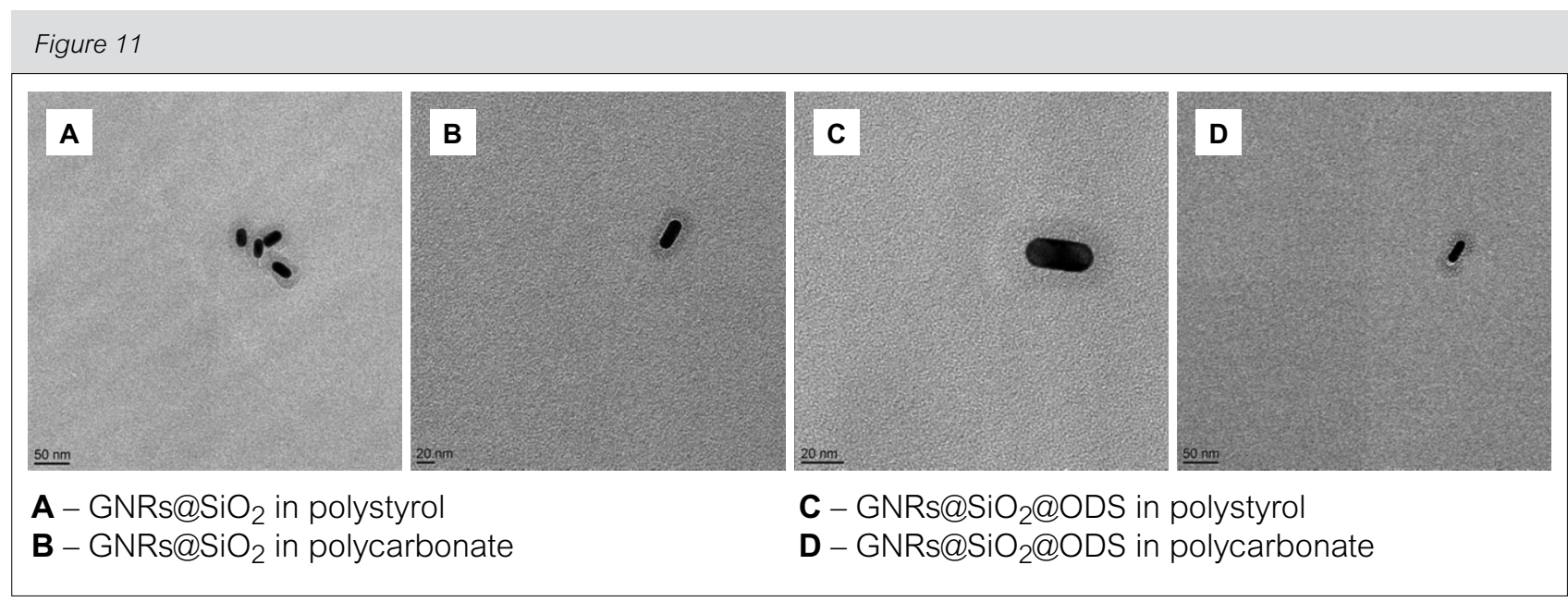

TEM images of the GNRs@SiO ${ }_{2}$ and GNRs@SiO $@ O D S$ with AR 4.2 inserted in polystyrol and in polycarbonate by extrusion. The image A shows a small agglomerate of GNRs@SiO in polystyrol. However, it should be noted that in this sample most of the rods are well dispersed as shown for samples $B, C$ and $D$

\section{Conclusions}

During this study, the well known silver-assisted seeded method was used to prepare GNRs with $A R \sim 4.2$. The colouring strength of the rods prepared during this study was demonstrated to be 4 to 8 times higher than those of commercial pigments. The GNRs were coated with a mesoporous silica shell of around $15 \mathrm{~nm}$ using a single-step Stöber approach and further functionalised with ODS. The silica shell greatly enhances the thermal stability of the rods up to at least 260 ${ }^{\circ} \mathrm{C}$. GNRs@SiO ${ }_{2}$ and GNRs@SiO $@$ ODS were successfully dispersed in both polystyrol and polycarbonate by extrusion. These plastics were greatly coloured using a tiny amount of GNRs. However, the colour of the rods evolves during extrusion due to the mesoporosity of the silica shell. Interestingly, the ODS functionalisation stabilises the colouration of the rods upon extrusion and drying in air.

\section{About the authors}

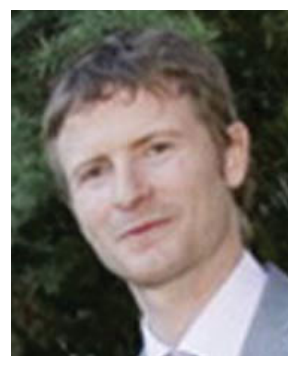

Cyrille Gautier is a R\&D chemist at Metalor Technologies in Neuchâtel, Switzerland. He has obtained his PhD degree at Neuchâtel University in the Surface Spectroscopy and

Nanoscience Laboratory. His research activities include the production, the characterization and the applications of nanomaterials based on noble metals.

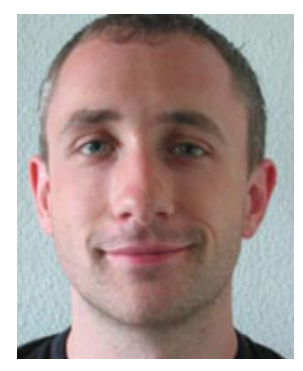

Alastair Cunningham is a $\mathrm{PhD}$ student at the University of Heidelberg working on novel means of functionalising and organising gold nanorods. Previously, he completed a Masters in Micro and Nanotechnology at the University of Neuchâtel in Switzerland and obtained an MSc in Chemistry from the University of Strathclyde in Glasgow.

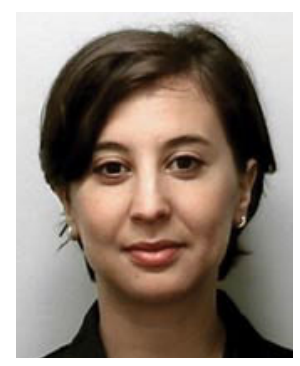

Lynda Si-Ahmed, PhD, is the Nanotech manager at Metalor Technologies SA in Neuchâtel, Switzerland. The Nanotech Unit is dedicated to the development and manufacturing of Precious Metal Nanoparticles.

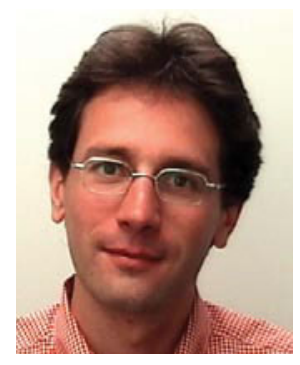

Gilles Robert, PhD, is the manager of the Technology Product Department at Metalor Technologies SA in Neuchâtel, Switzerland. The Technology Product Department is dedicated to the development and market introduction of innovations based on Metalor core competences in metallurgy and inorganic chemistry of precious metals. 


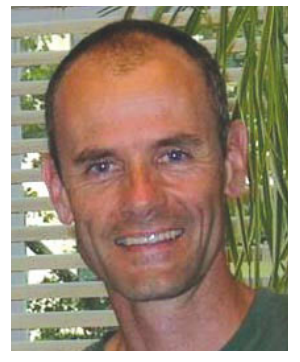

Thomas Bürgi obtained his $\mathrm{PhD}$ degree from the University of Bern, Switzerland. After a postdoc at MIT he made his habilitation thesis at ETH Zurich. In 2003 he moved to the University of Neuchâtel as a Professor of Physical Chemistry funded by the Swiss National Science Foundation. In 2008 he was appointed as a professor of Physical Chemistry at the University of Heidelberg. He is working in the fields of in situ spectroscopy of solid - liquid interfaces, photocatalysis and the preparation, characterization and application of nanomaterials.

\section{Acknowledgements}

We are grateful to the World Gold Council GROW program for financial support of this project. We cordially thank Prof. Clemens Holzer from INKA institute and Michèle Vlimant from Neuchâtel University for providing facilities and help in extrusion and ultramicrotomy, respectively.

\section{References}

1 C. Wang, J. Irudayaraj, Small 2008, 4, 2204

2 C.Z. Li, K.B. Male, S. Hrapovic, J.H.T. Luong, Chem. Commun. 2005, 3924

3 P.K. Sudeep, S.T.S. Joseph, K.G. Thomas, J. Am. Chem. Soc. 2005, 127, 6516

4 K.G. Thomas, S. Barazzouk, B.I. Ipe, S.T.S. Joseph, P.V. Kamat, J. Phys. Chem. B 2004, 108, 13066

5 H. Ding, K.T. Yong, I. Roy, H.E. Pudavar, W.C. Law, E.J. Bergey, P.N. Prasad, J. Phys. Chem. C 2007, 111, 12552

6 L. Tong, Y. Zhao, T.B. Huff, M.N. Hansen, A. Wei, J.X. Cheng, Adv. Mater. 2007, 19, 3136

7 N.M. Lawandy, A.Y. Smuk, J. Soc. Inf. Display 2005, 13, 755

8 J.W.M. Chon, C. Bullen, P. Zijlstra, M. Gu, Adv. Funct. Mater. 2007, 17, 875

9 P. Zijlstra, J.W.M. Chon, M. Gu, Opt. Express 2007, 15, 12151

10 J. Pérez-Juste, I. Pastoriza-Santos, L.M. Liz-Marzán, P. Mulvaney, Coord. Chem. Rev. 2005, 249, 1870

11 X. Xu, T. Gibbons, M.B. Cortie, Gold Bull. 2006, 39, 156

12 C.J. Orendorff, C.J. Murphy, J. Phys. Chem. B 2006, 110, 3990

13 I. Freestone, N. Meeks, M. Sax, C. Higgitt, Gold Bull. 2007, 40, 270

14 N.R. Jana, L. Gearheart, C.J. Murphy, Adv. Mater. 2001, 13, 1389

15 B. Nikoobakht, M.A. El-Sayed, Chem. Mater. 2003, 15, 1957

16 T.K. Sau, C.J. Murphy, Langmuir 2004, 20, 6414

17 M.Z. Liu, P. Guyot-Sionnest, J. Phys. Chem. B 2005, 109, 22192
18 L.I. Elding, A.B. Gronong, Acta. Chem. Scand. A 1978, 32 867

19 J. Pouradier, M. Coquard, J. Chim. Phys. 1966, 63, 1072

20 I. Pastoriza-Santos, J. Perez-Juste, L.M. Liz-Marzan, Chem. Mater. 2006, 18, 2465

21 C.A.J. Foss, G.L. Hornyak, J.A. Stockert, C.R. Martin, J. Phys. Chem. 1992, 96, 7497

22 Y.Y. Yu, S.S. Chang, C.L. Lee, C.R.C. Wang, J. Phys. Chem. B 1997, 101, 6661

23 F. Kim, J.H. Song, P. Yang, J. Am. Chem. Soc. 2002, 124, 14316

24 P.K. Jain, K.S. Lee, I.H. El-Sayed, M.A. El-Sayed, J. Phys. Chem. B 2006, 110, 7238

25 B. Nikoobakht, J. Wang, M.A. El-Sayed, Chem. Phys. Lett. 2002, 366, 17

26 H. Liao, J.H. Hafner, Chem. Mater. 2005, 17, 4636

27 S.S. Chang, C.W. Shih, C.D. Chen, W.C. Lai, C.R. Chris Wang, Langmuir 1999, 15, 701

28 M. Zhu, G. Qian, Z. Wang, M. Wang, Mat. Chem. and Phys. 2006, 100, 333

29 K. Dick, T. Dhanasekaran, Z. Zhang, D. Meisel, J. Am. Chem. Soc. 2002, 124, 2312

30 W. Stöber, A. Fink, E. Bohn, J. Colloid Interface Sci. 1968, 62, 26

31 I. Gorelikov, N. Matsuura, Nano Lett. 2008, 8, 369

32 S.O. Obare, N.R. Jana, C.J. Murphy, Nano Lett. 2001, 1, 601

33 J. Perez-Juste, M.A. Correa-Duarte, L.M. Liz-Marzan, Appl. Surf. Sci. 2004, 226, 137

34 C.J. Murphy, T.K. Sau, A.M. Gole, C.J. Orendorff, J. Gao, L. Gou, S.E. Hunyadi, T. Li, J. Phys. Chem. B 2005, 109, 13857

35 E.P. Barrett, L.G. Joyner, P.P. Halenda, J. Am. Chem. Soc. 2002, 73, 373

36 Y. Yin, Y. Lu, Y. Sun, Y. Xia, Nano Lett. 2002, 2, 427

37 C.T. Kresge, M.E. Leonowicz, W.J. Roth, J.C. Vartuli, J.S. Beck, Nature 1992, 359, 710

38 J.R. Sambles, Proc. R. Soc. London, Ser. A 1971, 324, 339

39 H.W. Sheng, K. Lu, E. Ma, Nanostruct. Mater. 1998, 10, 865

40 K.F. Peters, J.B. Cohen, Y.W. Chung, Phys. Rev. B 1998, 57, 13430

41 A.N. Goldstein, C.M. Echer, A.P. Alivisatos, Science 1992, 256, 1425

42 C.L. Cleveland, W.D. Luedtke, U. Landman, Phys. Rev. B 1999, 60, 5065

43 P. Buffat, J. P. Borel, Phys. Rev. A 1976, 13, 2287

44 J. P. Borel, Surf. Sci. 1981, 106, 1

45 M. B. Mohamed, K.Z. Ismail, S. Link, M.A. El-Sayed, J. Phys. Chem. B 1998, 102, 9370

46 El-Sayed A.M. Al-Sherbini, Colloids Surf. A 2004, 246, 61

47 H. Petrova, J.P. Juste, I. Pastoriza-Santos, G.V. Hartland, L.M. Liz-Marzan, P. Mulvaney, Phys. Chem. Chem. Phys. 2006, 8, 814

48 C. Wang, Z. Ma, Z. Su, Nanotechnology 2006, 17, 1819 